\title{
The Design of Ultrasonic Liquid Level Detection System Based on ARM
}

\author{
Ling Long, Bing Yi, Heng Xie \\ College of Electrical \& Information Engineering, \\ Southwest University for Nationalities, \\ Chengdu, 610041, China
}

\begin{abstract}
In order to detect the liquid level values in ordinary lives and industrial production easier, faster and more accurately, this system took ARM as the controller. The ultrasonic emitting and receiving circuit ensured the reliable ultrasonic transmission time. By proper conversion process of ARM, the data reflecting the actual liquid level could be obtained and be sent to TFT touch screen circuit to display the value and draw the variation curve of the liquid level. The experimental data had shown that the system was with reasonable range ability and had good measuring efficiency and precision.
\end{abstract}

Keywords: Liquid level detection; ultrasonic wave; ARM.

\section{INTRODUCTION}

With the development of electronic measurement technology, the ultrasonic ranging technology has been widely applied in people's work and lives[1]. It is a noncontact detection technology, not influenced by ray of light and the measured object color, and is more hygiene that other tools. It can work well in bad environments like moisture, dust, high temperature and corrosive gas. It needs less maintenance, has no pollution, is highly reliable and has longer life-span[2]. The ultrasonic ranging base on ARM controller, which has high processing speed and superior stability, has provided for the device reliable core processing system and high-speed real-time measuring characters, making full use of the advantages of ultrasonic ranging. Combining the wide application of ultrasonic detection and potential market outlook of ARM, this design is based on ARM and tries to dig the unique advantages of ARM in aspect of liquid level detection and market outlook.

\section{DESIGN OF SYSTEM HARDWARE STRUCTURE}

The control chip produces $40 \mathrm{KHz}$ control signal, which is amplified by a power amplifying signal to meet the requirement. Fig. 1 is the structural frame of ultrasonic liquid level detection device.

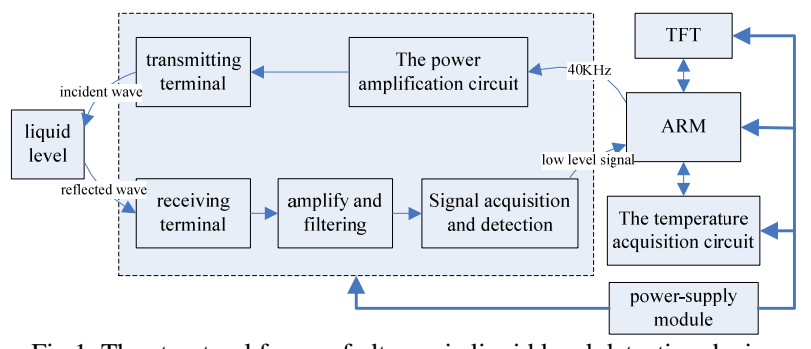

Fig.1. The structural frame of ultrasonic liquid level detection device
Make sure the amplified signal can drive the inverse piezoelectric sensor at the ultrasonic transmitting end to produce the ultrasonic wave. The transmitted ultrasonic wave then is reflected and the reflected wave is detected by the receiving end. The piezoelectric sensor at the receiving end correspondently produces electric signal with certain rule. Since this signal is weak and interfering, it needs to be amplified through pre-amplification to bigenough amplitude and then be processed through narrowband filter and signal comparison to output signals applicable and recognizable by the controller. The control chip will detect the applicable signals and calculate the measured distance and finally display the distance data and temperature data on TFT touch screen together.

\section{A. Ultrasonic transmitting module}

Multisim simulation diagram of ultrasonic transmitting end circuit is shown in fig. 2. The outer signal source produces $40 \mathrm{KHz}$ pulsing signal, which makes triode Q1 alternate switch producing V (OUT) alternate voltage and then drives the ultrasonic probe to send out $40 \mathrm{kHz}$ ultrasonic wave. The transmitting system is thus realized. Its working voltage is $12 \mathrm{~V}$. Q1common emitter configuration amplifies the signal. The selection of R1 and R2 is to obtain the driving voltage signal of proper amplitude.

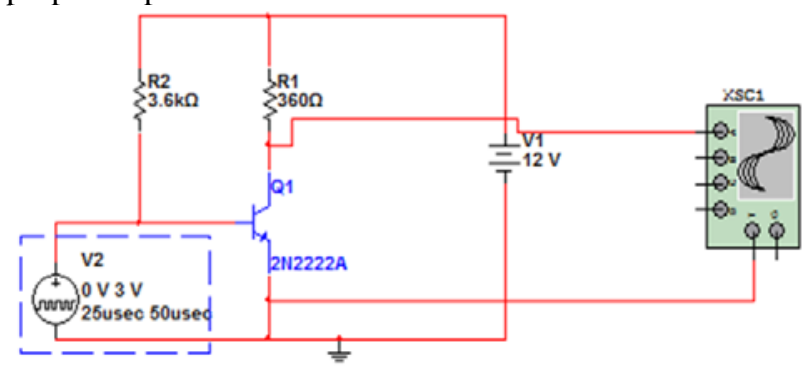

Fig.2. Multisim simulation diagram of ultrasonic transmitting end circuit

Fig. 3 is the driving signal oscillogram at the transmitting end. From this we can see that the wave form meets the requirement generally. Burrs occur in jump moments. Large amount of small clutters exits in high frequency. But the complete performance of the wave form reaches the design requirement. When the signal is applied to the transmitting end the ultrasonic transmitting probe, the clutters are absorbed by the ultrasonic sensor. The effects are good in actual circuit verification. 


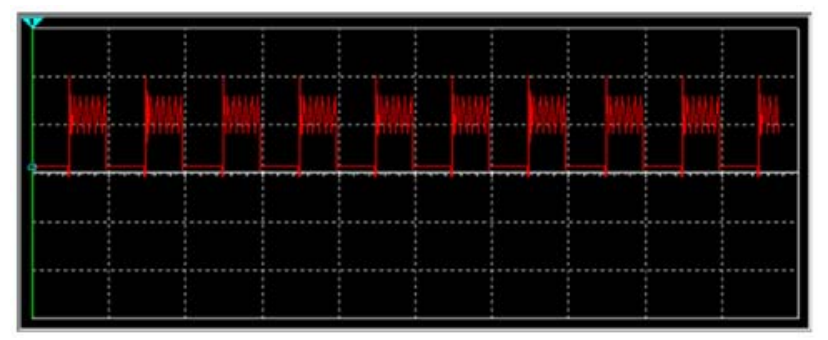

Fig.3. The driving signal oscillogram at the transmitting end

\section{B. Ultrasonic receiving module}

The ultrasonic receiving module is important to the whole design of hardware. It can amplify, filter, detect and compare the signals of the sensor at ultrasonic receiving end. This system adopts a type of ultrasonic transmitting module chip CX20106A produced by SONY. The chip has high frequency selecting ability that ensures the accurate signal frequency at the transmitting end. When CX20106A receives a $40 \mathrm{KHz}$ signal, a low level falling pulse will be produced at the 7th foot and the signal could be connected with the external interrupt pin of the control chip as the interrupt signal output. With the time span from occurring signal to receiving the interruption, the measured distance could be calculated. CX20106A has 8 foot single in-line plastic package, including automatic bias control circuit, preamplifier, limiting amplifier, band pass filter, peak detection, integral comparison and Schmidt shaping output circuit with a few external components[3,4]. It can complete all the functions of receiving and processing ultrasonic waves. The typical voltage is $5 \mathrm{~V}$ and the typical power is $9 \mathrm{~mW}$. Since CX20106A doesn't use inductor, it is free of interference of magnetic field. So it is easy to use and has less debugging troubles.

\section{SYSTEM PROGRAMMING}

The system programming includes the design of ultrasonic transmitting, receiving module program and data processing module program. The ultrasonic transmitting and receiving module is the core hardware module as well as the important software module of the system.

\section{A. Design of ultrasonic transmitting and receiving module program}

The flow chart of ultrasonic transmitting and receiving module program is shown in fig.4. First, through the clock divider, STM32 can produce $40 \mathrm{KHz}$ pulse and transmit the ultrasonic waves by driving the ultrasonic probe through the power amplification circuit and start the timer at the same time. When the ultrasonic wave is bounced back at reaching the liquid level, the ultrasonic receiving probe will transform the sound pressure to electric signal. A reasonable voltage could be got by amplifying the signal. The filtering circuit can select the useful signals of 40KHz. Compare the output pulses. When ALIENTEK MiniSTM32 detects echoes, the timer will be turned off and the time of ultrasonic transmission could be got[5]. Calculate with the ultrasonic sound speed in actual environment measured from DS18B20 and the distance from ultrasonic probe to liquid level could be got[6,7]. Then transform the result to the height of liquid level and finally translate the code and display on the touch screen.

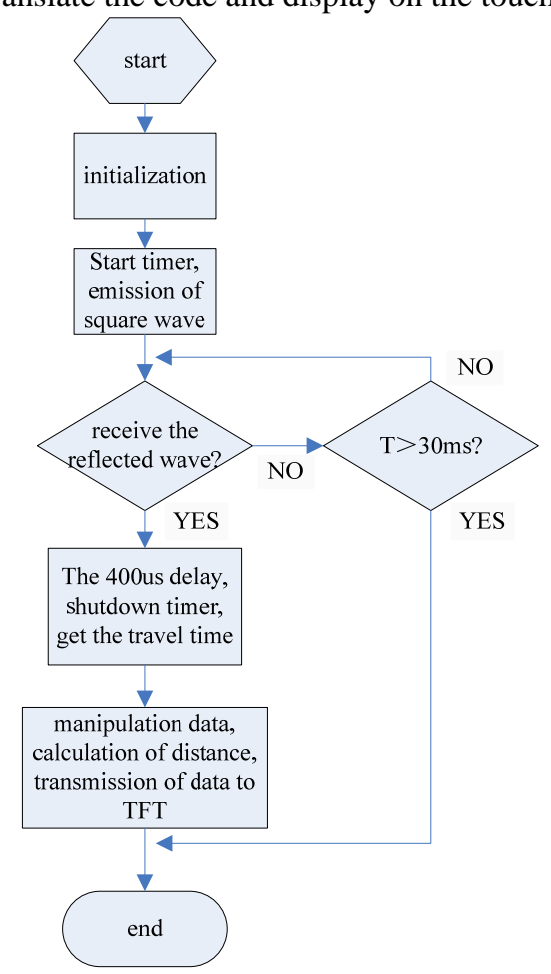

Fig.4. The flow chart of ultrasonic transmitting and receiving module program

\section{B. Design of data processing module program}

The data processing module is the core of this system software. The data of both temperature compensation module and ultrasonic transmitting and receiving module need to be processed by this module and then be sent to touch screen displaying module. The precision of data processing by this module directly influence the precision of measured results. So it is extremely important. First, the real time temperature of external environment $T$ obtained from temperature sensor is sent to the touch screen as the current temperature $T$. Then from the approximate formula $C=C 0+0.607 \times T^{\circ} C$, the real time ultrasonic velocity $C$ at the current temperature could be obtained and displayed on the touch screen. The ultrasonic travel time $\mathrm{t}$ could be got from ultrasonic transmitting and receiving module. With the formula $S=C * t / 2$ the height of liquid level or the Distance could be got. Finally, the touch screen will display distance.

\section{CONCLUSIONS}

By software simulation and hardware circuit test, we can see that the system has high performance price ratio, high stability and is easy in using. Within the liquid level 
range $20 \mathrm{~mm}-4.5 \mathrm{~m}$, we could get better measuring precision.

\section{ACKNOWLEDGEMENTS}

This work was financially supported by the Fundamental Research Funds for the Central Universities of Southwest University for Nationalities (NO. 2014NZYQN10).

\section{REFERENCES}

[1] Zhang Hai-Ying, Gao Yan-Li. The Research of Ultrasonic Ranging Technique. Instrumentation Technology,9,pp.58-60,2011.

[2] Li Peng. Application of Ultrasonic Ranging. Technology and Life, 23,pp.115,2010.

[3] Fang Zheng-Chao, Yao Shan-Xue. Design of ultrasonic ranging based on CX20106A.International Electronic Elements. 30, pp.151-153, 2012.

[4] Qing Wei, Yan Wen-Jun. Design of Ultrasonic Car Reversing Radar for Parking Based on CX20106A, Piezoelectrics \& Acoustooptics,33, pp.161-164, 2011.

[5] Wang Xiao. touch-screen experimentation based on ALIENTKE MiniSTM32 development board. PC Fan, 3, pp. 1-2, pp. 31, 2014.

[6] Zhang Jun. Smart Temperature Sensor DS18B20 and Its Application. Instrumentation Technology. 4, pp.68-70, 2010.

[7] Tang Kai-Jie, etc..Design of digital temperature collection and alarm system based on DS18B20.Transducer and Microsystem Technology,33, pp.99-102, 2014. 